\title{
ALGUNAS REFLEXIONES EN TORNO AL CARÁCTER NORMATIVO - VALORATIVO- DE LA ASIGNACIÓN DE RESPONSABILIDAD PENAL
}

\author{
Ilsse Carolina Torres Ortega ${ }^{1}$
}

\section{Resumen}

En este trabajo la autora presenta algunas reflexiones relacionadas con la asignación de responsabilidad penal. En primer lugar, realiza un análisis conceptual de la responsabilidad y un acercamiento a lo que implica la "asignación de responsabilidad". Luego, en segundo lugar, estudia el concepto de castigo en un intento por mostrar cómo la relación entre ambos conceptos (responsabilidad y castigo) podría ayudarnos a avanzar en un proyecto desafiante: el de dilucidar qué tipo de teoría debería guiar la asignación de responsabilidad penal. A lo largo de este artículo, se argumenta que tal asignación puede reconstruirse más sólidamente como una tarea normativo-valorativa a la luz de una filosofía práctica del Derecho penal. Finalmente, explica cómo esto ha de proyectarse a nuestros sistemas de responsabilidad.

Palabras clave: Responsabilidad, castigo, asignación de responsabilidad penal, normatividad

\section{INTRODUCCIÓN}

El presente trabajo pretende abordar la compleja cuestión de la asignación de responsabilidad penal. Para ello se distinguirán tres apartados, cada uno de ellos referido a un nivel de reflexión distinto que, en conjunto, pueden esclarecer algunos aspectos de la ya aludida complejidad. En primer lugar, se utilizará el aparato teórico-conceptual de H. L. A. Hart para explorar qué subyace a la afirmación de que una persona es responsable de una acción delictiva, así como qué subyace a la aparentemente obvia elección de sistemas de justicia que exigen la satisfacción de determinadas condiciones para considerar legítima la aplicación de un castigo.

Luego de las reflexiones anteriores, en la segunda parte de este trabajo se expondrán algunos apuntes de tipo metodológico acerca de qué tipo de teoría (meta-teoría) sería la más adecuada para tratar todas aquellas condiciones que exigimos han de ser satisfechas para

\footnotetext{
${ }^{1}$ Doctora en Derecho (Filosofía del Derecho) por la Universidad de Alicante. Profesora-investigadora en el Departamento de Estudios Sociopolíticos y Jurídicos del Instituto Tecnológico de Estudios Superiores de Occidente (ITESO) (Jalisco, México). Código ORCID: 0000-0002-5929-9137. Correo electrónico: torresilsse@iteso.mx.
} 
asignar responsabilidad penal. En este punto, se explorarán (y se defenderán) las siguientes tres afirmaciones: 1- La responsabilidad (entendida en su acepción de responsabilidad como sancionabilidad) y el castigo son conceptos de tipo normativo-valorativo -entendiendo normativo no en el mero sentido de referente a normas o dependiente de normas, sino en el sentido de normatividad que nos remite al razonamiento justificativo-; 2- entre tales conceptos -responsabilidad y castigo- existe una relación que es de tipo conceptual; 3- esta relación se pone de manifiesto al exigir una fundamentación racional de la asignación de responsabilidad penal y, por tanto, esta debe ser vista también como una tarea normativa (en el sentido que se acaba de señalar) que se desarrolla dentro del marco de una filosofía práctica del Derecho penal.

Finalmente, después de haber expuesto la necesidad de una teoría de asignación de responsabilidad penal que sea normativo-valorativa, se presentarán algunas reflexiones acerca de cómo tendría que ser tal teoría para poder considerarse, en efecto, normativa. En este nivel, se trata de un proyecto consistente en generar principios básicos generales (de ética normativa) que sirvan para revisar críticamente la justicia de nuestras prácticas jurídicopenales.

\section{RESPONSABILIDAD Y ASIGNACIÓN DE RESPONSABILIDAD}

Tal y como indica Guillermo Lariguet, nos ocupamos de los conceptos por buenas razones: "No lo hacemos por prurito especulativo, sino porque queremos comprender los anclajes referenciales de nuestros conceptos que no son otros que estados de cosas, conductas, prácticas que parecen estar impregnadas, o al menos 'circundadas', por nuestros conceptos" (2016, 234). De ahí la conveniencia de explorar conceptos como el de responsabilidad (y un poco más adelante, el de castigo) y, además, hacerlo a través de un autor como Hart, quien junto a otros, por supuesto- supo mostrar la envergadura de los problemas de vaguedad y 
ambigüedad del lenguaje jurídico ${ }^{2}$, al tiempo que renegó de la "pedantería" analítica que aspiraba a recoger la totalidad de la realidad jurídica en su actividad de análisis ${ }^{3}$.

\section{EL CONCEPTO DE RESPONSABILIDAD. LA RESPONSABILIDAD COMO SANCIONABILIDAD}

En general, parece bastante claro que el concepto de responsabilidad forma parte del vocabulario básico del Derecho. Más allá del Derecho, como afirma Isabel Lifante, se trata de uno de los pilares básicos de la filosofía práctica general, ya que, a través de éste se alude a la posición del ser humano como sujeto activo en el mundo (perspectiva ética deontologista) y a la relevancia moral de las consecuencias de nuestras acciones (perspectiva consecuencialista): "Aunando ambas intuiciones podríamos decir que considerar a alguien como agente, es decir, como sujeto responsable, implica reconocer, por un lado, que tiene el control de su actuación y, por otro, que asume o debe asumir las consecuencias de sus actos" (Lifante, 2017, 109).

Lo anterior constituye el punto de partida para apreciar la importancia que tiene la responsabilidad en contextos de normas que establecen marcos de referencia respecto a cómo debemos comportarnos, así como la atribución de responsabilidad para aquellos supuestos en los que tales marcos son inobservados. Sin embargo, antes de desarrollar esto último, es conveniente ahondar un poco más en la cuestión de la responsabilidad.

Actualmente es casi un lugar común que cualquier reflexión que intente ir más lejos en el análisis de la responsabilidad comience aludiendo a la diversidad de sentidos de la expresión señalada por H. L. A Hart en el post-script "Responsibility and Retribution" de la obra Punishment and Responsibility. En este trabajo, en lugar de intentar determinar un

\footnotetext{
${ }^{2}$ Como bien se sabe, Hart sostuvo que términos como el de responsabilidad, derecho subjetivo, deber, etc., constituían términos anómalos; estas anomalías, de acuerdo con Hart, son básicamente tres: en primer lugar, la diversidad de casos en los que se usan los términos jurídicos, ya que esto complica el encontrar un principio que indique su campo de aplicación; en segundo término, el hecho de que no hay una contrapartida empírica; y, en tercer lugar, su uso en el contexto jurídico no representa ni describe nada. Desde Definition and Theory in Jurisprudence -su lección inaugural en Oxford en 1953- Hart anunció la relevancia de la filosofía analítica del lenguaje ordinario en las controversias clásicas sobre los conceptos jurídicos. Anterior a esta lección, había presentado The adscription of Responsibility and Rights, en el cual, fuertemente influenciado por Austin, Hart había planteado también la importancia del análisis del lenguaje en la reflexión iusfilosófica, pero proponiendo que el análisis de los conceptos jurídicos podía realizarse a través de los actos del habla.

${ }^{3}$ Como muestra de ello lo que expresa Hart antes de enunciar los distintos sentidos de responsabilidad: "Espero que al dibujar estas líneas divisorias, y en la exposición que sigue, haya evitado las arbitrarias pedanterías de la sistemática clasificatoria, y que mis divisiones seleccionen y clarifiquen las principales, aunque no todas, las variedades de responsabilidad cuyas referencias son constantemente realizadas, explícita o implícitamente, por filósofos morales, juristas, historiadores y personas comunes" (Hart, 2008b, 212. La traducción es mía).
} 
concepto que lograra dar cuenta de las características esenciales del uso del término ${ }^{4}$, Hart elucidó cuatro sentidos de "responsabilidad" que resultaban de gran importancia en el ámbito jurídico-práctico ${ }^{5}$ : (1) la responsabilidad derivada de un rol, (2) la responsabilidad causal, (3) la responsabilidad como capacidad y (4) la responsabilidad como sancionabilidad ${ }^{6}$ (liabilityresponsibility) (Hart, 2008b, 212) ${ }^{7}$.

\begin{abstract}
${ }^{4}$ Al final de Punishment and Responsibility, Hart agregó algunas notas a los capítulos que componen el libro. Respecto al capítulo IX -en el que se ocupa de los distintos sentidos de responsabilidad- el autor aclaró que no intentó identificar una característica unificadora que explique las diferentes aplicaciones del término. Pese a lo anterior, destaca que los principales sentidos de responsabilidad están conectados con la idea de "responder", de tener que dar respuestas o rendir cuentas de algo. Así, incluso llama la atención respecto a que el significado original de la palabra "responder" (del latín respondere) estaba relacionado con responder no a preguntas, sino a contestar o refutar una acusación que, de ser afirmada, tenía como consecuencia la imposición de sanciones como el castigo.

${ }^{5}$ Hart intentó mostrar la amplia gama de diferentes, aunque conectadas, ideas que son abarcadas por las expresiones de responsabilidad, responsable y responsable de, tal y como estas expresiones son utilizadas en el Derecho. Para ilustrar esta variedad enunció la siguiente historia sobre el capitán de un barco: Como capitán del barco, X era responsable de la seguridad de los pasajeros y de la tripulación. Sin embargo, durante su último viaje el capitán se embriagó cada noche y fue responsable de la pérdida del barco con todo lo que éste tenía a bordo. Se rumoreó que estaba loco, pero los médicos consideraron que es responsable de sus acciones. Durante todo el viaje se comportó irresponsablemente, y varios incidentes en su carrera demuestran que él no era una persona responsable. El capitán siempre mantuvo que las tormentas excepcionales fueron las responsables de la pérdida del barco, pero en los procesos jurídicos seguidos contra él fue encontrado penalmente responsable por su conducta negligente, y civilmente fue encontrado jurídicamente responsable de la pérdida de vidas y bienes. El capitán aún vive y es moralmente responsable
\end{abstract} de las muertes de muchas mujeres y niños (Hart, 2008b, 211).

${ }^{6}$ Este sentido de responsabilidad suele ser traducido de diferentes maneras. Optar por una traducción "más literal" como la de sujeción es adecuado cuando pretende enfatizarse la posición de la persona y no tanto al tipo de reacción que se sigue de dicha sujeción. Por otra parte, optar por un término como el de sancionabilidad pretende subrayar, precisamente, las consecuencias normativas seguidas de la responsabilidad. Dado que en esta ocasión interesa, centralmente, esto último, se utilizará tal expresión para referirse a este sentido. Adicionalmente, es necesario señalar que dentro de este sentido de responsabilidad como sancionabilidad hay que distinguir la responsabilidad como sancionabilidad jurídica (legal liabilityresponsibility) y la responsabilidad como sancionabilidad moral (moral liability-responsibility).

${ }^{7}$ El primero de estos sentidos, la responsabilidad como rol, se refiere al uso de esta expresión para hacer referencia a los deberes y obligaciones que posee una persona por ocupar un cargo o rol distintivo en una organización o actividad social; estos deberes tienen que ver con la promoción del bienestar y de ciertas metas de la organización que se los confiere. Este sentido también se relaciona con el uso de expresiones del tipo $\mathrm{X}$ es responsable o $\mathrm{X}$ tiene un comportamiento responsable para representar a una persona dispuesta a tomar seriamente sus deberes y hacer el mayor esfuerzo posible para su cumplimiento. La responsabilidad causal presenta cierto uso de la expresión en el que es posible sustituir frases del tipo $\mathrm{X}$ fue responsable de por palabras como causado o producido, u otras expresiones causales que hacen referencia, descriptivamente, a consecuencias o resultados que pueden atribuirse no solo a los seres humanos, sino también a sus acciones u omisiones, así como a cosas, condiciones y eventos. Sin embargo, esta acepción tiene también una dimensión normativa, ya que suele ser una condición de sancionabilidad el hecho de que entre la acción del sujeto y el daño (el delito) exista cierta conexión causal, es decir, que el segundo sea efecto del primero. El sentido de responsabilidad como capacidad se suele representar con la expresión X es responsable de sus acciones, la cual se refiere a las capacidades que pueden ser atribuidas, prima facie, a cualquier ser humano adulto, tales como el entendimiento, el razonamiento y el control de la conducta. Que una persona posea este tipo de capacidades es una condición de eficacia del Derecho (para poder influir en la conducta de las personas), pero también representa una exigencia moral para la sancionabilidad; y es que tal parece que muy pocos estarían dispuestos a defender la corrección moral de un sistema de justicia que permitiera sancionar a individuos privados de estas capacidades básicas. Por lo que corresponde a la responsabilidad como sancionabilidad, en el contexto jurídico hablar de responsabilidad normalmente es algo relacionado con la transgresión de normas jurídicas que requieren a los individuos hacer o no hacer 
No se analizarán de manera independiente estos sentidos, puesto que, a efectos de lo que se pretende plantear a continuación, es el sentido de responsabilidad como sancionabilidad el que ha de ocupar nuestra atención. Es este sentido el que permitirá plantear la cuestión de qué tipo de teoría ha de ser aquella que rija la asignación de responsabilidad penal, así como las implicaciones que derivan de esta primerísima reflexión.

Tal y como señala Karin Boxer, el tratamiento que hizo el autor de la responsabilidad como sancionabilidad es el más detallado de la variedad de sentidos que indicó, pero también es el menos claro (Boxer, 2015, 38). Con este sentido, Hart hace referencia directa a la transgresión de normas jurídicas (concretamente, normas de conducta) que requieren a los individuos actuar -o no actuar- de determinada forma (dimensión regulativa); esto sería así, porque, de acuerdo con otras normas, se juzga la conducta llevada a cabo por el individuo (dimensión valorativa), siendo probable que quien infringe una disposición de este tipo sufra la imposición de un castigo (responsabilidad penal, punibilidad), o bien quede sujeto a tener que compensar a las personas que ha dañado (responsabilidad civil), o a ambas consecuencias. En los dos casos se dice coloquialmente que la persona "tiene que pagar por lo que hizo" ${ }^{8}$.

No obstante, el propio Hart añade algo más que resulta realmente interesante en su planteamiento. Hart intentó mostrar que las expresiones abstractas de responsabilidad (responsibility) y de responsabilidad como sancionabilidad (liability), si bien parecen ser equivalentes en el contexto jurídico-sancionador, no lo son. La expresión "X es jurídicamente responsable por $Y$ (un daño o una acción)" no siempre es equivalente a " $X$ es jurídicamente responsable (sancionable) para ser castigado o tener que pagar una compensación por $Y^{\prime \prime}$; esto último parece estar referido a algo más específico. El planteamiento de Hart consiste en que esta última expresión nos remite a la cuestión de qué requisitos han de verse satisfechos

determinadas conductas, ya que, de acuerdo con otras normas jurídicas, quien infringe una disposición de este tipo queda sujeto a ser castigado por su conducta, o bien, sujeto a tener que compensar a quienes ha dañado (Hart, 2008b, 215).

${ }^{8}$ No obstante, nuestros sistemas jurídicos pueden ir más lejos y establecer que alguien puede quedar sujeto a "pagar" por lo que hizo alguien más (un empleado, por ejemplo), o incluso a pagar una compensación sin que medie la acción u omisión de alguien más (por ejemplo, por una maquinaria defectuosa cuya condición no podría haber sido descubierta). Estos casos se conocen como responsabilidad vicaria y, aunque están vetados en los sistemas de Derecho penal, no por ello hay que dar por superada la consideración sobre por qué suele ser exigido para castigar a alguien que esta persona haya hecho por sí misma la acción prohibida por la ley.

${ }_{9}^{9}$ Precisamente, como la responsabilidad y la sancionabilidad son distinguibles, tiene sentido decir que debido a que una persona es jurídicamente sancionable por una acción, ésta es susceptible de ser castigada por ello (Hart, 2008b, 222). 
para sancionar a alguien, lo que vendría a incluir las condiciones de responsabilidad ${ }^{10}$. Es necesario señalar que de aquí en adelante únicamente se hará referencia a la responsabilidad penal (punibilidad).

\section{LA ASIGNACIÓN DE RESPONSABILIDAD PENAL}

En este orden de ideas, la responsabilidad como sancionabilidad constituye la idea base de lo que se entenderá por asignación de responsabilidad. Así, atribuir responsabilidad penal a un agente ha de entenderse como una actividad de imputación relacionada con la contrastación entre la acción (u omisión) de un sujeto y ciertos criterios de responsabilidad que legitiman que éste sea susceptible de sufrir un castigo. Esto es, se trata de una práctica guiada por valores (la justicia en la distribución de castigos).

Siguiendo a Hart, entonces, afirmar la sancionabilidad de alguien no conlleva a la mera afirmación de que existió una transgresión a normas jurídicas. Esto es, que alguien sea jurídicamente sancionable no implicaría únicamente que llevó a cabo una acción prohibida, sino que se han satisfecho determinados criterios de responsabilidad que el sistema jurídico concreto ha establecido. Hart sostiene que estos criterios normalmente se agrupan en tres clases: 1- condiciones mentales o psicológicas; 2- criterios de conexión causal u otras formas de conexión entre el acto y el daño; y 3- criterios sobre las relaciones entre el agente y el individuo susceptible de ser castigado o de pagar por los actos de otros ${ }^{11}$ (Hart, 2008b, 217 y 218). De hecho, esto es lo que lleva a Hart a defender, al final de Punishment and Responsibility, que el sentido de sancionabilidad es el sentido primario, en tanto que los demás sentidos constituyen condiciones de la sancionabilidad ${ }^{12}$.

Lo anterior puede verse con mayor claridad si se atiende a la distinción trazada por Pablo Larrañaga entre reglas de responsabilidad (que aluden a las reglas que establecen las condiciones para imputar sanciones a los individuos, distintas de la violación de la norma de conducta), sistemas de responsabilidad (que se refieren a las normas de conducta y las reglas

\footnotetext{
10 Con lo anterior, al parecer Hart establece una diferencia de alcance entre las condiciones de responsabilidad y las condiciones de sancionabilidad (que serían más amplias e incluirían a las cuestiones de responsabilidad).

${ }^{11}$ Estos criterios se refieren a la posibilidad de responsabilidad directa (el sujeto responde por sus propios actos) y la vicaria (el sujeto responde por los actos de otra persona). Sin embargo, como ya se había mencionado, en el Derecho penal liberal solo se admite la primera.

${ }^{12}$ Sin embargo, precisamente porque estas condiciones son dependientes de otra reflexión acerca de qué justifica la imposición de la sanción, bien puede suceder que haya un sistema de responsabilidad que no exija la satisfacción del requisito de responsabilidad como capacidad y que pueda considerarse aún legítimo (aunque no sería el caso penal).
} 
de responsabilidad) ${ }^{13}$, y los juicios de responsabilidad (que aluden al resultado de contrastar una acción con un sistema de responsabilidad) (Larrañaga, 2004, 198-201). Así, es posible sostener que en la propuesta de Hart los sentidos antes aludidos funcionan como reglas de responsabilidad y, por lo tanto, forman parte de las condiciones que han de verse satisfechas en los juicios concretos de asignación.

De esta forma, cuando lo que se pretende es atribuir responsabilidad penal a un individuo -y así considerarle apto para soportar la consecuencia jurídica que establece la norma- nunca se hace referencia a una verificación simple acerca de que una persona llevó a cabo una acción transgresora; esto es, no se trata de un mero problema de calificación normativa. La responsabilidad como sancionabilidad ha de entenderse íntimamente comprometida con la satisfacción de otras condiciones que esbozan la legitimidad de la sanción -en este caso, el castigo- y, por ello, es posible afirmar que comparte la pretensión de justificación de la sanción y es, por tanto, normativo-valorativa; además, esta misma idea es la que permite ir dimensionando la relación existente entre la responsabilidad y el castigo.

Ahora bien, lo anterior, por sí solo, es una mera trivialidad, puesto que nos coloca delante de un amplio abanico de posibilidades. Y es que lo relevante de esta cuestión radica, precisamente, en el contenido de las reglas de responsabilidad. El primer paso para avanzar en ello, sin embargo, debe ser reconocer que estas reglas, para poder ser dotadas de contenido, no pueden ser entendidas en un sentido meramente descriptivo, sino que tienen una dimensión que es valorativa, puesto que se pretende que las mismas estén justificadas y no sean productos de la arbitrariedad.

Por ejemplo, de los diversos tipos de condiciones de sancionabilidad señalados por Hart parecen ser especialmente relevantes aquellas que exigen ciertas aptitudes psíquicas presentes en el individuo (básicamente, lo que entendemos por imputabilidad), así como los estados mentales bajo los cuales se lleva a cabo la acción ${ }^{14}$. La satisfacción de requisitos de este tipo, se supone, nos autorizaría a asumir al individuo como agente moral responsable. Detrás de la exigencia de que el individuo posea ciertas capacidades subyace una ya clásica discusión acerca de que la responsabilidad, necesariamente, implica un ejercicio de libertad

\footnotetext{
${ }^{13}$ En abstracto y en concreto. Condiciones ideales de sancionabilidad y el caso concreto.

${ }^{14}$ Esto es evidente en la configuración de la teoría del delito que, precisamente, ha tenido sus principales modificaciones en atención a los "elementos subjetivos". Por ejemplo, a partir de la sistemática finalista se trasladó el análisis del dolo y la culpa al elemento del tipo y quedó reservado para la culpabilidad el estudio de la capacidad. En general, la teoría del delito ha seguido distintas sistemáticas: la teoría del causalismo naturalista (Franz von Liszt y Ernst von Beling); la teoría del causalismo valorativo (Edmund Mezger), la teoría del finalismo (Hans Welzel) y la teoría del funcionalismo (Claus Roxin con una versión moderada y Günther Jakobs con una radical).
} 
(libre albedrío) y que, por tanto, solo se puede ser responsable de actos voluntarios ${ }^{15}$. Por supuesto, no interesa plantear aquí el problema del determinismo ${ }^{16}$, por lo que simplemente se señala que la idea de dignidad que hemos asumido como ingrediente fundamental de la concepción liberal del individuo y de la sociedad establece que los seres humanos deben ser tratados de acuerdo a sus decisiones, intenciones o manifestaciones de consentimiento (Nino, 1989, 287). Esto supone, entonces, que tratar con dignidad a las personas implica tomar lo anterior como antecedentes válidos de juicios de responsabilidad que involucran consecuencias jurídicas.

Lo que se pretende mostrar es que la sola elección de un sistema jurídico que tome en cuenta las condiciones mentales de las personas es ya una cuestión valorativa: creemos que hay razones que justifican la afirmación de que un juicio de responsabilidad que tome en cuenta lo anterior es mejor que uno que no lo haga. Muestra de ello es que consideremos lo anterior una conquista de nuestros sistemas jurídico-penales y que, incluso, se contemplen en nuestros códigos penales diversas causas de excusa y de justificación, así como atenuantes ${ }^{17}$.

\footnotetext{
${ }^{15}$ A pesar de la trascendencia del tema, Liborio Hierro indica que la afirmación o negación del libre albedrío como fundamento de la responsabilidad penal es, al mismo tiempo, una cuestión clásica y una cuestión marginal en la ciencia del Derecho penal: "parece que todos los penalistas se vieran forzados a tomar posición respecto a esta cuestión para, a continuación, reconocer que su solución por una parte les excede y por otra les resulta indiferente" (Hierro-Sánchez Pescador, 1989, 561).

${ }_{16}$ El tema del determinismo y las controversias filosóficas que existen en torno a éste es demasiado complejo. En general, corresponde a una concepción que niega que los actos de voluntad y las decisiones de las personas sean relevantes, entendiéndolos como simples manifestaciones de otros hechos - por ejemplo, sus rasgos biológicos. Hay diversas clases de determinismo, por ejemplo, el determinismo ético (los seres humanos no pueden eludir conscientemente el bien y si actúan de otra forma es por ignorancia o compulsión), el teleológico (dios tiene un conocimiento previo de los actos humanos, por lo que los hombres no pueden actuar de modo diverso), el lógico (la verdad de una proposición es algo atemporal, por lo que no se puede alterar su valor de verdad), el psicológico (las acciones están determinadas causalmente por deseos, motivaciones, etc.), el físico (las acciones están determinadas causalmente por fenómenos mecánicos o químicos que pasan dentro del organismo), etc. Todos tienen en común la idea de que los seres humanos no poseen una genuina libertad para actuar y tomar decisiones, y que, por tanto, no pueden ser considerados responsables, moral ni jurídicamente, de sus actos. El problema del libre albedrío ha estado presente en la historia de la filosofía desde el inicio y, dadas las dificultades -o como algunos sostienen, la imposibilidad- de demostrar tanto la tesis determinista como la de la existencia de libertad, en décadas más recientes se han defendido diversas versiones de la postura compatibilista que pretende llegar a un punto de conciliación. La versión más difundida del compatibilismo sostiene que aun si resultara cierta la tesis determinista -cualquiera de ellas, pese a que son muchas y distintas entre sí- esto sería compatible con la libertad de acción. Basándose en los planteamientos de Peter Strawson, se señala que debe ser una razón de peso el hecho de que la vida social (muchas de nuestras prácticas sociales más importantes), tal y como la conocemos, se basa en la idea de que la voluntad, las decisiones o el consentimiento de las personas deben tomarse como antecedentes válidos de consecuencias normativas.

${ }^{17}$ Para Hart, justamente, la preocupación por las condiciones mentales se traduce jurídicamente en la previsión de excusas, justificaciones y atenuantes. La admisión de circunstancias eximentes (o que disminuyen) de responsabilidad penal es una parte crucial del compromiso de los sistemas penales por distribuir el castigo de una forma justa que garantice que los individuos no serán tratados como medios para la consecución del fin preventivo del castigo. Hart consideró que probar la transgresión voluntaria de una regla era una especie de autorización moral que se garantizaba, en la medida de lo posible, al prever estas figuras.
} 
¿QUÉ TIPO DE TEORÍA DE ASIGNACIÓN DE RESPONSABILIDAD PENAL?

Con lo anterior, se ha intentado poner de manifiesto que la determinación del contenido de las reglas de responsabilidad no parece ser una cuestión que deba dejarse en manos de la arbitrariedad; todas ellas han de ser formuladas atendiendo a la finalidad de que la atribución de responsabilidad sea justa. Ser responsable de un estado de cosas que a la luz del ordenamiento jurídico es disvalioso -la responsabilidad-sancionabilidad-, significa ser una persona que puede ser justificadamente castigada.

Lo que se plantea a continuación es, precisamente, qué subyace a esta pretensión de justicia o, más concretamente, en qué han de fundamentarse dichas reglas para suponer que genuinamente atienden a tal pretensión, considerando que cuestión aparte es si, de hecho, ésta es satisfecha.

Pues bien, se sostiene que el carácter normativo-valorativo de la responsabilidad penal está determinado por el propio carácter normativo-valorativo del castigo y que es esto lo que, además, permite establecer entre ambos conceptos una relación conceptual. Para ello, es conveniente primeramente aclarar qué se está entendiendo por castigo, para después plantear el interrogante de porqué habría de incorporarse conceptualmente a un sentido de responsabilidad como el anteriormente bosquejado.

Es necesario señalar que esta relación conceptual puede defenderse en un sentido más elemental. Por ejemplo, Garzón Valdés, hablando sobre enunciados de responsabilidad retrospectivos condenatorios -el enunciado de responsabilidad como enunciado de imputación que lleva a considerar: (1) un estado de cosas como incorrecto o indeseable, (2) un agente que dio lugar a tal estado de cosas y que puede ser reprochado por sus actos y, (3) una autoridad autorizada para formular tal enunciado y exigir que el autor rinda cuentas-, señala que la imputación de autoría formulada en tal enunciado lleva a un estado de cosas con implicaciones normativas (el castigo), lo que permite sostener que la relación entre responsabilidad y castigo es conceptual o lógica, ya que hablar de responsabilidad en estos casos implica hablar de castigos (Garzón Valdés, 1996, 259-286). Esto quiere decir que, en tanto que la activación del concepto de responsabilidad penal genera la activación del concepto de castigo, hay una relación conceptual o lógica. Si bien se asume plenamente lo anterior, se intentará ir un poco más lejos sobre los vínculos que conectan a ambos conceptos.

\section{EL CONCEPTO DE CASTIGO}


A lo largo de la historia de las ideas se han ofrecido definiciones que pretenden identificar los criterios de aplicación del término castigo -entre otras finalidades, para distinguirlo de otras sanciones jurídicas ${ }^{18}$; ; sin embargo, muchas de ellas parecen esconder un propósito mucho más ambicioso y, sin embargo, cuestionable: ofrecer los criterios de legitimidad de esta institución, ya sea descubriendo la esencia imperecedera del castigo ${ }^{19}$, o bien, estableciendo una barrera definicional que, artificiosamente, determine en qué casos estamos ante algo que puede ser llamado castigo y que, por definición, estaría justificado ${ }^{20}$.

A los efectos de una mejor comprensión del castigo, es conveniente partir de alguna definición que sea lo más descriptiva y exhaustiva posible, al menos en el sentido de que no contenga un pronunciamiento sobre su legitimidad.

Una caracterización que aporta mucho como primer acercamiento al concepto es la señalada por el propio Hart, quien propuso la siguiente caracterización del caso estándar del castigo $^{21}:$ 1) Debe involucrar dolor $u$ otras consecuencias normalmente consideradas no placenteras, o desagradables; 2) tiene que ser por una transgresión a reglas jurídicas; 3) tiene que ser aplicado a un real o supuesto delincuente por su transgresión; 4) tiene que ser administrado intencionalmente por seres humanos distintos del delincuente y; 5) debe ser impuesto y administrado por una autoridad constituida por el sistema jurídico en contra del cual se cometió la transgresión (Hart, 2008 a 4 y 5).

No obstante, este ejercicio de análisis conceptual ha de ser matizado. La definición de Hart es esclarecedora y, además, permite reparar en que la cuestión conceptual del castigo no puede ser utilizada para zanjar el problema de su justificabilidad -no incluye como propiedad definitoria una que implique que el castigo está, por definición, justificado-; sin

\footnotetext{
${ }^{18}$ Esta cuestión resulta de especial relevancia en un tema como este, puesto que el castigo, como problema filosófico tradicional, se ha abordado precisamente sobre la base de que hay una diferencia cualitativa entre el castigo y la sanción.

${ }^{19}$ Hay muchos teóricos que, aunque abiertamente no abrazan una visión conceptualista, implícitamente continúan viendo en la tarea conceptual un fin en sí mismo que permite comprender en su totalidad una institución. En el caso particular del castigo, muchas controversias se han estancado en la discusión de un concepto que logre dar cuenta de las condiciones de la responsabilidad penal, del delito y de la propia pena, cuando lo que sucede es que gran parte de la determinación de cada uno de estos conceptos depende de los principios y conclusiones normativas que desarrollemos previamente.

${ }^{20} \mathrm{La}$ barrera definicional se refiere a la situación en que una persona evade el tener que dar argumentos sobre la fundamentación de un caso concreto como caso de castigo, aduciendo que, por definición, no puede estar justificado porque no se dan los elementos definitorios suficientes para hablar propiamente de castigo. ${ }^{21}$ Hart señaló, además del caso central del castigo, algunos casos sub-estándar o secundarios: a) castigos por infracciones de reglas jurídicas impuestas o administradas por personas distintas de los funcionarios (sanciones descentralizadas), b) castigos por infracciones a reglas u órdenes no jurídicas (castigos en la familia o en la escuela), c) castigos indirectos o colectivos a algún miembro de un grupo social por sanciones hechas por otros sin la previa autorización, estímulo, control o permiso, y d) castigo de personas (distintas de las incluidas en (c)) que no son ni de hecho ni supuestamente transgresores (Hart, 2008, 5). Considerando estos casos secundarios el autor evidenció que no obviaba el uso ordinario del término, sino que, simplemente, optaba por centrar su análisis en el contexto jurídico.
} 
embargo, el concepto tiene, además de propiedades descriptivas, otras que son de carácter valorativo (relacionadas, precisamente, con la justificación), las cuales no se eluden con la simple precisión de un caso central que no incluya tal propiedad. Y es que en el caso del castigo habría que seguir una estrategia distinta, una dinámica de sucesivas aproximaciones dialécticas entre el concepto y su justificación ${ }^{22}$ : así, partimos de los conceptos de la primera aproximación para discutir sobre ellos (utilizándolos) y formular los principios justificativos del castigo; a través de esta discusión depuramos los conceptos, identificando sus propiedades relevantes y proyectando una teoría sustantiva sobre el castigo; una vez que definimos más precisamente los conceptos, utilizamos el nuevo esquema conceptual para volver a someter a escrutinio los principios justificativos. Este proceso de ajustes mutuos sigue mientras continúe la discusión sobre la fundamentación del castigo. Para avanzar en ello se apunta la siguiente reflexión.

En nuestros sistemas jurídicos se prevén sanciones negativas que autorizan la privación de bienes que son especialmente valiosos; estas sanciones son, precisamente, las que identificamos como castigos. Como cualquier sanción jurídica, se trata de un acto coercitivo llevado a cabo intencionalmente por alguien autorizado para ello e implica la privación de bienes de quien ha cometido una transgresión jurídica. Los castigos, sin embargo, no implican cualquier privación de bienes, sino la vulneración de aquellos que consideramos objetivamente superiores. Es este el presupuesto normativo-valorativo -pues depende de un juicio de valor respecto a la jerarquía de los bienes- que nos permite plantear la cuestión del castigo.

Esta normatividad también se manifiesta en que la institución del castigo jurídico envuelve una pretensión de justificación. Privar a alguien de un bien es algo prima facie indebido que, o se justifica como una excepción a dicho principio, o bien, será simplemente un acto de fuerza. En el Derecho es posible afirmar que la privación de bienes pretende ser un acto legítimo (por ello se establecen sistemas de responsabilidad), esto es, una excepción justificada al principio de que no está permitido dañar a otros ${ }^{39}$. Si la privación de bienes que se autoriza implica la lesión de bienes tan valiosos como pueden ser la vida o la libertad, tanto más fuerte habrá de ser la pretensión de justificación y tanto más estrictas las exigencias en

\footnotetext{
${ }^{22}$ El análisis y la reconstrucción conceptual, entendidos como instrumentos de clarificación teórica, por sí solos no aportan nada significativo sobre la justificación del castigo. Aunque es preciso mantener la distinción metodológica entre el discurso descriptivo y el discurso justificativo, ambos discursos están conectados cuando abordamos una cuestión como la del castigo, perteneciente al mundo de la praxis.
} 
que haya de expresarse (condiciones ideales de sancionabilidad o reglas de responsabilidad $)^{23}$.

El carácter valorativo del concepto determina que la reconstrucción del mismo vaya ligado a la cuestión de su fundamentación. Esto es, es necesario indagar sobre qué nos autoriza a imponer una sanción jurídica que afecte bienes especialmente valiosos para poder determinar propiedades de aplicación del concepto. Esta reflexión acerca de la justificación del castigo, además, da lugar al marco de referencia para la propia aplicabilidad del concepto normativo-valorativo de responsabilidad, puesto que aquello que justifique la imposición de castigos es lo que habrá de procurarse a través de los sistemas de responsabilidad (normas de conducta y reglas de responsabilidad). Es por esto que se considera que, en un contexto de razonamiento práctico, hay una relación conceptual entre la responsabilidad y el castigo.

\section{LA FUNDAMENTACIÓN DEL CASTIGO Y DE LA RESPONSABILIDAD}

Tal y como se mencionó al inicio de este trabajo, el Derecho, entendido como práctica social normativa - de acuerdo con una concepción argumentativa o post-positivista del Derecho-, posee una dimensión regulativa que lleva a establecer una serie de pautas de conducta que establecen deberes y prohibiciones a los ciudadanos. Dichas pautas de conducta, sin embargo, son derivadas de ciertos fines y valores que el Derecho aspira a alcanzar y a desarrollar ${ }^{24}$, lo que supondría que son marcos de referencia válidos-legítimos a la luz de los principios que han de regir el espacio de lo intersubjetivo- no solo para la cuestión de cómo debemos comportarnos, sino también para el juicio de aprobación o desaprobación de la conducta realizada. A esto último, bajo esta concepción del Derecho, se le identifica como dimensión valorativa, puesto que ya no se trata de una cuestión prospectiva, sino de un ejercicio retrospectivo que evalúa la conducta ya hecha. Sucede, además, que en este contexto normativo las normas deónticas o regulativas establecen consecuencias jurídicas para el caso de su transgresión (ilícitos típicos), las cuales tienen, esencialmente, la finalidad de motivar la conducta de los ciudadanos y ofrecerles una razón para actuar de acuerdo con aquellas

\footnotetext{
${ }^{23}$ Infligir sufrimiento deliberado a otros nos obliga a ofrecer razones morales que respalden, en determinadas situaciones, causarles daño. Estas razones nunca pueden darse por supuestas. Aunque esta no es la única institución dentro del Derecho que permite imponer algún tipo de daño o privación a las personas, la pena exige un análisis mucho más sofisticado por las formas en que se materializa tal daño.

${ }^{24}$ Esto se corresponde con la idea del Derecho como práctica social. Tal y como señala Atienza, en esta idea podemos identificar tres componentes: (1) una concepción dinámica del Derecho; (2) la distinción de una dimensión autoritativa y otra axiológica -gozando esta última de cierta preeminencia-; y (3) la necesaria vinculación de la práctica jurídica con valores morales objetivos $(2017,35)$.
} 
conductas que favorezcan la convivencia social y la satisfacción de intereses generales e individuales. En este sentido, Victoria Roca indica:

\begin{abstract}
"Es importante ver la relación funcional o instrumental que existe entre los juicios ex post acompañados de sanciones institucionalizadas y la dimensión de guía de conducta que tiene el Derecho. Y es que se pretende que la amenaza de una pena, una condena al pago de una cantidad, el reproche moral, el juicio de ilicitud jurídica, sean incorporados al razonamiento por parte de los sujetos que en un futuro deliberen acerca de cómo actuar. Por ejemplo, en el caso de las conductas sancionadas (sanción negativa), se trata de que los individuos incorporen tanto razones vinculadas con el valor (razones morales), como también con los propios intereses (razones prudenciales) para abstenerse de volver a llevar a cabo conductas que son contrarias a las normas jurídicas en las que se ha de sustentar la convivencia (prevención especial y general) (Roca Pérez, 2015, $82)$ ".
\end{abstract}

De lo anterior, es posible identificar que, respecto de las sanciones negativas en general, en la teoría del Derecho se ha admitido una justificación, predominantemente, consecuencialista -prevencionista-, aunque, claro está, limitada por razones deontológicas; de ahí la exigencia, por ejemplo, de que solo un agente moral puede ser responsable. El debate sobre la fundamentación del castigo es similar a las ideas antes señaladas, con la diferencia de que la tensión entre ambas concepciones morales - deontologismo y consecuencialismose ha acentuado tanto que el planteamiento básico del castigo supone la oposición retribucionismo vs. prevencionismo ${ }^{25}$.

No se desarrollarán los aspectos sustantivos de esta dicotomía; pero, sí ha de señalarse que el debate sobre la fundamentación del castigo no puede limitarse a ésta ${ }^{26}$, sino que ha configurarse como un problema de ética normativa cuya respuesta ha de venir entretejida con la teoría normativa que fundamenta la fuerza justificativa del Derecho. Esto quiere decir que no nos ha de interesar qué es, como objeto contemplativo, el castigo, sino cómo justificar su existencia dentro de nuestros ordenamientos jurídicos y cómo se traduce esto en la aplicación de castigos. Dicha reflexión ha de sustentarse en aquello que, de acuerdo con los principios

\footnotetext{
${ }^{25}$ En términos muy básicos, la concepción retribucionista enfatiza el merecimiento moral del individuo que ha causado el mal; tal merecimiento es razón necesaria y suficiente para castigar, además de ser la medida de su pena, puesto que el sufrimiento merecido debe ser, de alguna forma, proporcional al mal causado. La justificación de la pena, bajo esta concepción, no puede sostenerse en las consecuencias que se obtengan de su aplicación, al considerar que, de otra forma, el individuo corre el riesgo de ser instrumentalizado. La concepción prevencionista, por su parte, justifica la pena en las buenas consecuencias que pueden obtenerse con su imposición. El castigo debe ser un medio efectivo para evitar daños futuros, puesto que el daño cometido ha sido ya realizado y es imposible de "retribuir"; por tanto, el castigo no debe ser una forma de venganza institucionalizada, sino tomar en cuenta las consecuencias de su aplicación.

${ }^{26}$ Sobre esta dicotomía es interesante tener en cuenta lo indicado por Mitchell Berman: "En ocasiones se insinúa que las discusiones filosóficas acerca del castigo están plagadas de disensos radicales. Pero esto es una exageración. En al menos un asunto, el consenso se aproxima a la unanimidad: el castigo necesita de una justificación. Muy a menudo, este principio constituye la primera premisa del teórico del castigo" $(2008,1)$.
} 
de justicia y de moralidad social que se admiten para juzgar acciones e instituciones, permite considerar como justa la institución del castigo y legitimar su aplicación a personas $\operatorname{concretas}^{27}$.

Dado que la práctica punitiva es una de las formas más controvertidas que tiene el Derecho para procurar el cumplimiento de sus disposiciones -la imposición de un castigo penal es, seguramente, la expresión más manifiesta de la coacción en general, un elemento central del Derecho-, la justificación moral del castigo es una condición necesaria de la justificación moral del Derecho en general y del Derecho penal en particular (Nino, 2001, 428). ${ }^{28}$.

Ahora bien, cualquiera podría reprochar que esto aún parece estar muy alejado de la actividad concreta de asignar responsabilidad penal a los individuos y que es, más bien, un razonamiento iusfilosófico contemplativo sin implicaciones en la configuración del sistema de justicia penal. Sin duda, esto sería cierto si no se insiste en la importancia de llevar esta discusión del terreno puramente teórico-descriptivo al práctico o normativo crítico, lo cual implica una concepción de esta disciplina como filosofía del Derecho práctica que tiene entre sus objetos de interés el desarrollo de las reflexiones de ética normativa (o de ética sustantiva). Es decir, se trata de generar herramientas conceptuales y tesis sustantivas desde la perspectiva interna a la propia práctica del Derecho, con el fin de que ese esclarecimiento, desde el nivel de la fundamentación, no solo reconstruya conceptualmente la práctica jurídica, sino que también aporte herramientas adecuadas para que la misma sea repensada y conducida a la luz de adecuados criterios de justicia. En esto radica la verdadera normatividad de la asignación de responsabilidad penal.

\section{HACIA UNA GENUINA TEORÍA NORMATIVA -VALORATIVA- DE ASIGNACIÓN DE RESPONSABILIDAD PENAL}

Ahora bien, una vez hecho todo este recorrido es necesario regresar a la cuestión de cómo asignar justificadamente responsabilidad penal y, más importante aún, abordar cómo

\footnotetext{
${ }^{27}$ Respecto a esto último es muy importante tener presente la distinción lógica entre justificar una práctica y justificar una acción particular que cae dentro de ella. El nivel de reflexión sobre la justificación de la institución se centra en los principios que subyacen a la admisión de penas en nuestros ordenamientos deliberar sobre las razones que legitiman a esta institución tal y como se encuentra plasmada en el Derecho positivo-, mientras que, en otro nivel, reflexionar sobre la justificación de su aplicación se refiere a la asignación de responsabilidad penal a sujetos concretos.

${ }^{28}$ Esto, en realidad, es contrario a lo que se sigue en la dogmática jurídico-penal que centra el estudio de la cuestión penal en el delito; tanto es así que prácticamente toda la reflexión penal es reconducible a la teoría del delito y sus elementos.
} 
habría de ser una teoría sobre la misma que incorpore lo anterior en el contexto de nuestros sistemas jurídicos.

Y es que, ciertamente, contamos ya con un aparato institucional que rige la asignación de responsabilidad. El propósito de estas páginas no es sugerir que este aparato debe cuestionarse desde sus raíces. Solo se pretende subrayar la necesidad de que el mismo se analice a la luz de aquellos presupuestos que consideramos que hacen que un castigo esté justificado. Se tratará entonces de exponer dichos presupuestos y de someterlos a deliberación, formulando principios generales que, posteriormente, puedan ayudar a mejorar el sistema positivo y, con ello, la labor de los operadores jurídicos. Esto, ciertamente, solo puede ser un proyecto de ética normativa para el ámbito del Derecho penal.

Hablar de un proyecto integral de adjudicación de responsabilidad penal, lógicamente, no es nada nuevo. La dogmática jurídico-penal, a través de la teoría general del delito (su concepción estratificada), históricamente ha buscado sistematizar las condiciones que definen al delito, las cuales, de seguirse, permitirían asignar adecuadamente responsabilidad penal a los individuos. Tal y como señala Winfried Hassemer, la teoría de la imputación en Derecho penal tiene como tarea principal la cuestión de cuándo y bajo qué condiciones se puede establecer una relación, que sea penalmente relevante, entre una persona y un suceso para aplicar una sanción penal $(1999,157)$. La crítica que se ha venido haciendo desde hace bastante tiempo es que este proyecto es insatisfactorio porque no ha reconocido abiertamente el carácter normativo de los principios básicos que sostiene, y esquiva la tarea de proveer una justificación moral de los mismos.

No se quiere aquí cometer el error de simplificar la importante e ingente tarea de la dogmática, ni tampoco entrar a desarrollar las críticas que en el mencionado sentido se han dirigido a la misma. El propósito es únicamente dejar apuntado que tales críticas han de ser tomadas en serio, en tanto que expresan algunas insuficiencias que impiden tomar el modelo de la dogmática como modelo de teoría normativa-valorativa de asignación de responsabilidad penal.

Para avanzar en este modelo, es necesario articular una propuesta que siga una metodología distinta. En este sentido, se destaca el proyecto de Carlos S. Nino. La propuesta de este autor consistió en defender algunos principios generales -resultantes de su reflexión sobre la justificación del castigo- como la base normativa de una teoría para la adjudicación de responsabilidad penal. De acuerdo con Nino, tal base estaría integrada por (1) el principio de protección prudencial de la sociedad, (2) el principio de intersubjetividad del Derecho penal (anti-perfeccionista), (3) el principio de asunción de la pena y (4) el principio de 
antijuridicidad (inicialmente llamado principio de enantiotelidad). En términos muy resumidos, esto quiere decir que la pena es siempre un mal que se inflige a ciertos individuos -también es un mal para la sociedad-que solo puede estar justificado si se puede demostrar

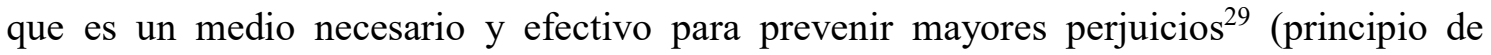
protección prudencial); que las conductas por las que se puede castigar a un individuo solo deben ser conductas tendentes a prevenir daños a terceros y no conductas que dañen únicamente al propio agente (principio de intersubjetividad); que para no utilizar a un individuo, castigándolo en beneficio de la sociedad, hace falta que éste haya consentido la asunción de la responsabilidad penal (principio de asunción de la pena); y que la conducta del agente efectivamente haya causado el daño (o lo haya puesto en peligro objetivo) que a través de las leyes se está intentando prevenir (principio de enantiotelidad). Es muy importante mencionar que, además, estos principios básicos derivan de los principios más generales de autonomía, inviolabilidad y dignidad de la persona.

No se pretende hacer aquí una reconstrucción de la teoría del autor; tampoco comprometerse con todo el contenido de la misma. Lo que sí interesa destacar es el camino seguido por Nino: 1- En primer lugar, plantea el problema de la justificación de la pena como un sucedáneo de cierta concepción de la sociedad -el Estado democrático liberal (liberalismo igualitarista) -y como problema central de la fundamentación del Derecho penal que, como tal, está detrás de las dificultades que se presentan en la práctica para determinar la sancionabilidad de las personas. 2- A partir de esta reflexión, formula una serie de principios básicos que ofrecen una propuesta tentativa de cómo puede justificarse el castigo (los problemas de ética práctica no tienen "soluciones" como tales) que tiene en cuenta no solo las teorías clásicas, sino los ideales liberales del sistema jurídico. 3- A través de estos principios, conforma una teoría normativa de adjudicación de responsabilidad penal que permite formular criterios de corrección para evaluar las disposiciones y la práctica vigentes (Nino, 1989).

Tomando este recorrido como inspiración, se sostiene que un proyecto en los términos indicados incorpora la normatividad de los distintos elementos que intervienen en los juicios de responsabilidad.

\section{CONSIDERACIONES FINALES}

\footnotetext{
${ }^{29} \mathrm{El}$ autor dice que esto implica los tres requisitos clásicos del estado de necesidad: el perjuicio que se quiere evitar es mayor que el que se causa, la pena es efectiva para evitar tales perjuicios y no hay medida más económica en el daño social que provoca.
} 
Sin duda, pensar en la asignación de responsabilidad no es un asunto sencillo. A lo largo de este trabajo se ha intentado poner de manifiesto la necesidad de indagar, por una parte, en la responsabilidad y, por otra, en las sanciones que suelen ser impuestas como resultado de una actividad que conocemos como asignación de responsabilidad. En este trabajo el análisis se ha centrado en aquellas sanciones que constituyen castigos, al ser, seguramente, el caso más problemático de aquellas medidas coactivas previstas en nuestros ordenamientos jurídicos que implican privación de bienes. Se ha intentado mostrar cómo este caso, precisamente por ser uno de los más complicados, nos permite observar una serie de problemas relacionados con la normatividad. Por una parte, la responsabilidad, en su acepción de sancionabilidad, nos remite al cumplimiento de una serie de condiciones que funcionarían como guías en la asignación de responsabilidad y, por otra parte, la reflexión sobre aquello que legitima al castigo nos permite dotar de contenido dichas condiciones. Esto, a su vez, tiene como propósito, precisamente, el de asignar justificadamente responsabilidad penal e imponer -cuando así corresponda-, también justificadamente, un castigo a un sujeto en particular. Aquello que, según la reflexión crítica al respecto, consideremos que justifica la imposición de castigos constituye aquello que debe ser procurado a través de los sistemas de responsabilidad (normas de conducta y reglas de responsabilidad).

SOME REFLECTIONS ON THE NORMATIVE -VALORATIVE-CHARACTER OF THE CRIMINAL LAW ADJUDICATION

\begin{abstract}
In this paper, the author presents some related reflections on Criminal Law adjudication. Firstly, she undertakes a conceptual analysis of "responsibility", and an approach to the idea of "responsibility adjudication". Then, secondly, she studies the concept of "punishment" in an attempt to show how the relation between the concept of responsibility and the concept of punishment might help us to take steps forward in a challenging project: that of elucidating which sort of theory ought to guide Criminal Law adjudication. Throughout the work, it is argued that Criminal Law adjudication is to be more soundly reconstruct as a normative task under the light of a Practical Philosophy of Criminal Law. Finally, she explains how this is to be projected into our responsibility systems.
\end{abstract}

Keywords: Responsibility, punishment, Criminal Law Adjudication, Philosophy of Law, Philosophy of Criminal Law.

\title{
REFERENCIAS
}

ATIENZA, Manuel. Filosofía del Derecho y transformación social, Madrid: Trotta, 2017. 
BOXER, K. Hart's Senses of Responsibility en Hart on Responsibility (pp. 30-46), Nueva York: Palgrave MacMillan, 2014.

DUFF, R. A. Responsabilidad y punibilidad en el Derecho penal: El legado de H.L.A. Hart: Filosofía jurídica, política y moral, Madrid: Marcial Pons, 2012, 145-168.

DWORKIN, Ronald. Justicia para erizos, México: Fondo de Cultura Económica, 2014.

GARDNER, John. Hart y Feinberg sobre responsabilidad en El legado de H.L.A. Hart: Filosofía jurídica, política y moral, Madrid: Marcial Pons, 2012, 169-193

GARZÓN VALDÉS, Ernesto. El enunciado de responsabilidad. Doxa: Cuadernos de filosofía del Derecho, número 19: 259-286.

GONZÁLEZ LAGIER, Daniel. Las paradojas de la acción. Una introducción a la teoría de la acción humana desde el punto de vista del Derecho y de la filosofía, Madrid: Marcial Pons, (2013).

GONZÁLEZ LAGIER, Daniel. Hechos y acciones en el Derecho en Conceptos básicos del Derecho (pp. 65-80), Madrid: Marcial Pons, 2015.

HART, H. L. A. Prolegomenon to Principles of Punishment en Punishment and Responsibility: Essays in the Philosophy of Law, segunda edición, Oxford: Oxford University Press, 2008a, pp. 1-27.

HART, H. L. A. Postscript: Responsibility and Retribution en Punishment and Responsibility: Essays in the Philosophy of Law (pp. 210-237), segunda edición, Oxford: Oxford University Press, 2008 b.

HART, H. L. A. The Morality of the Criminal Law. Two Lectures, Israel: The Hebrew University of Jerusalem, 1965

HART, H. L. A. Law, Liberty and Morality. Stanford: University Press, 1963.

HASSEMER, W. Persona, mundo y responsabilidad, Valencia: Tirant Lo Blanch,1999. 
HIERRO SÁNCHEZ-PESCADOR, Liborio. Libertad y Responsabilidad Penal. Anuario de derecho penal y ciencias penales, 1989 , pp. 561-70.

LARIGUET, Guillermo. La investigación filosófica: ¿Análisis conceptual versus análisis histórico?”. Revista Praxis Filosófica Nueva serie, número 42, 2016, pp. 219-244.

LARRAÑAGA MONJARAZ, Pablo. El concepto de responsabilidad, México: Editorial Fontamara, 2004.

LIFANTE VIDAL, Isabel. Responsabilidad en el desempeño de funciones públicas. Anuario de Filosofía del Derecho, número 13, pp. 99-124, 2017.

LIFANTE VIDAL, Isabel . "Ignorancia de la ley y seguridad jurídica”. Teoría y derecho: Revista de pensamiento jurídico. No. 18: 16-33, 2015.

NINO, Carlos Santiago: Fundamentos de Derecho penal. Los escritos de Carlos S. Nino. Compilado por Gustavo Maurino, Buenos Aires: Editorial Gedisa, 2008.

NINO, Carlos Santiago. Introducción al análisis del derecho, Buenos Aires: Editorial Astrea, (2001)

NINO, Carlos Santiago. Subjetivismo y objetivismo en el Derecho Penal. Revista Jurídica. Universidad Autónoma de Madrid, número 1: 47-82, 1999.

NINO, Carlos Santiago. Ética y Derechos Humanos. Un ensayo de fundamentación, Editorial Ariel, Barcelona, 1989.

NINO, Carlos Santiago. Los límites de la responsabilidad penal: una teoría liberal del delito, Barcelona: Editorial Astrea, 1980.

MIR PUIG, Santiago. Introducción a las bases del Derecho penal, Argentina: Editorial B de F, segunda reimpresión, 2003. 
MOORE, Michael S. Causalidad y responsabilidad. Un ensayo sobre derecho, moral y metafísica, Madrid: Marcial Pons, 2011.

MUÑOZ CONDE, Francisco y GARCÍA ARÁN, Mercedes. Derecho Penal, Parte General, Octava edición, Valencia: Tirant lo Blanch, 2010.

ROCA PÉREZ, Victoria. Ilícito, responsabilidad y sanción en Conceptos básicos del Derecho, Madrid: Marcial Pons, 2015, pp. 81-112.

STRAWSON, Peter F. Libertad y resentimiento (y otros ensayos), Barcelona: Ediciones Paidós, 2012.

Trabalho recebido em 18 de janeiro de 2019 Aceito em 17 de fevereiro de 2019 